\title{
EEG PATTERN RECOGNITION: Application to a Real Time Control System for Android-Based Mobile Devices
}

\author{
Liliana Gutiérrez-Flores, Carlos Avilés-Cruz, \\ Juan Villegas-Cortez, and Andrés Ferreyra-Ramírez \\ Universidad Autónoma Metropolitana, Azcapotzalco. Departamento de Electrónica, \\ San Pablo Xalpa No.180, Col. Reynosa Tamaulipas, CP 02200, México, D.F. \\ \{ligf, caviles, juanvc, fra\}@correo.azc.uam.mx
}

\begin{abstract}
This paper describes a new EEG pattern recognition methodology in Brain Computer Interface (BCI) field. The EEG signal is analyzed in real time looking for detection of "intents of movement". The signal is processed at specific segments in order to classify mental tasks then a message is formulated and sent to a mobile device to execute a command. The signal analysis is carried out through eight frequency bands within the range of 0 to $32 \mathrm{~Hz}$. A feature vector is conformed using histograms of gradients according to 4 orientations, subsequently the features feed a Gaussian classifier. Our methodology was tested using BCI Competition IV data sets I. For "intents of movements" we detect up to $95 \%$ with 0.2 associated noise, with mental task differentiation around $99 \%$. This methodology has been tested building a prototype using an Android based mobile telephone and data gathered with an EPOC Emotive headset, showing very promising results.
\end{abstract}

Keywords: EEG Pattern Recognition, Self-Paced Control, BCI application, Mental Tasks Differentiation.

\section{Introduction}

A Brain Computer Interface (BCI) is a system that enables communication between brain and computer. For the last two decades, there have been a great interest using Electro-Encephalographic (EEG) signals on applications in order to help people with motor disabilities, such as amyotrophic lateral sclerosis [1] or spinal cord injury [2]. There are works implementing EEG signal processing, such as Tanaka et al. [3, which used left and right thinking to control the direction of an electric wheel chair; and Müller et al. 2] which used motor imagery movements to control prosthesis for the upper extremities. Recently, control applications have been developed for other purposes, for example: differentiation of six limb movements was used to control the Khepera robot [4; exploration, communication or monitoring space applications were improved with BCI technologies [5]; a BCI integrated to computer vision system was used to prioritize the interests of a person in different images [6]; and the differentiation of right 
and left imagery movements were used to control a video-game 7]. Our vision consists of people using thoughts to control mobiles in their daily lives. A lot of issues need to be solved, mainly concerning the usability of the EEG signal acquisition devices, but also with the effective differentiation of the mental task on real time, the efficient processing of the EEG signal over portable computers and of course wireless communications.

Synchronous or asynchronous approaches have been used to process the EEG signals. Synchronous is useful only for offline characterization and analysis of the signal; for online control, an asynchronous approach is needed. Plenty of work has to do with the fact of enabling self-paced control, the work of Borisoff et al. 8] was developed for enabling a switch to be activated with a movement of a finger, achieving a detection of $80 \%$; and Faradji et al. 9] processed data from five mental tasks in a self-paced fashion with $70 \%$ of detection, but in some cases without noise. Feature extraction is a very important stage, the more properly built the features, the more efficient results we got from the classifier, even if it is a simple one. Feature vectors have been conformed on a great variety of forms, from the work of Keirn et al. [10] which used left-right asymmetric ratio and spectral density with $90 \%$ of classification results with a Quadratic Bayesian classifier; adaptive autoregressive parameters and event related synchronization/desynchronization of the $\mu$ and $\beta$ rhythms where used by Pfurtscheller et al. 11, [11, [2]; Zhang et. al used power spectral entropy 12 achieving $90 \%$ of classification with a time-variable linear classifier; wavelet transform was used by Qin et al. [13] and Bostanov [14, the latter used a linear discriminant analysis to got $84 \%$ of classification, and Sun used energy difference with a support vector machine achieving $90 \%$ [15].

In the present work an EEG pattern recognition methodology and a prototype of one application in a real time control system of a mobile device are presented. The major functional blocks are shown in Fig[1. As the ongoing EEG signal is received, it must be continuously analyzed, we can not know in advance at what time the user is going to perform a mental task with the intention of executing a command, so we need to detect that precise time. In order to classify a mental task and correlates it to a command, the signal is processed in a window around the time detected in the previous stage. A feature vector is built over this window and then it is fed to a statistical parametric classifier (in our case a Gaussian classifier is used). The rest of the paper is organized as follows: In Section 2 our EEG pattern recognition methodology proposal is detailed; the evaluation data are shown in Section 3 . experimental results and prototype are explained in Section 4 and finally conclusions and further work are presented in Section 5.

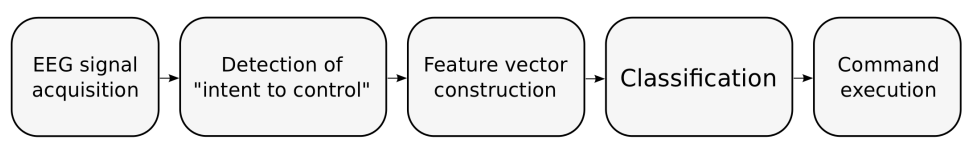

Fig. 1. Major functional blocks of the real time control system 


\section{EEG Pattern Recognition Methodology Proposal}

\subsection{Time-Frequency Pre-processing}

The whole input EEG signal is conformed by $N$-separated signal, where $N$ represents the number of available channels. The signal is processed over onesecond windows. Lets call $S^{k}$ the signal for any $k$-window (1), $M$ represents the number of samples per second.

$$
\mathbf{S}^{k}=\left(\begin{array}{ccccc}
s_{1,1}^{k} & \ldots & s_{1, j}^{k} & \ldots & s_{1, M}^{k} \\
\vdots & & \vdots & & \vdots \\
s_{i, 1}^{k} & \ldots & s_{i, j}^{k} & \ldots & s_{i, M}^{k} \\
\vdots & & \vdots & & \vdots \\
s_{N, 1}^{k} & \ldots & s_{N, j}^{k} & \ldots & s_{N, M}^{k}
\end{array}\right) .
$$

Since the EEG signal is highly non-stationary, the shift between one window and the next consists of only one sample. A new window is conformed for each sample after the first second of the signal elapsed. The mean of the signal for each channel $\overline{S_{i}^{k}}$ is subtracted from every $S_{i}^{k}$ row to eliminate the offset and produce $\widetilde{S_{i}^{k}}$. The spectral power $P_{i}^{k}$ for each channel is calculated using the short-Fourier transform $(\mathscr{F})$ with a Hanning window using (2) and subsequently the result is multiplied by its complex conjugate (3).

$$
\begin{gathered}
\mathscr{F}\left\{\widetilde{S_{i}^{k}}\right\}=\sum_{n=-\infty}^{\infty} \widetilde{S_{i}^{k}} W_{\text {Hanning }}[n] \boldsymbol{e}^{-j \omega n} . \\
P_{i}^{k}=\mathscr{F}\left\{\widetilde{S_{i}^{k}}\right\} \cdot \mathscr{F}^{*}\left\{\widetilde{S_{i}^{k}}\right\} .
\end{gathered}
$$

Low frequencies, between 0 and $32 \mathrm{~Hz}$, associated to delta $(\delta)$, theta $(\theta)$, alpha $(\alpha)$ and beta $(\beta)$ rhythms are the most important part of the spectral power $P_{i}^{k}$. Eight bands of frequency are conformed from $P_{i}^{k}$ as follows: $(0-4],(4-$ 8], $(8-12],(12-16],(16-20],(20-24],(24-28],(28-32]$. A single value $P_{i j}^{k}$ associated to each frequency band is calculated using (4), $i=1, \ldots, N$ denotes the channel, $j=1, \ldots, 8$ denotes the band of frequency, and $p_{i, m}^{k}$ the $m$-value of $P_{i}^{k}$, for $m=1, \ldots, 32$.

$$
P_{i, j}^{k}=\frac{1}{4} \sum_{m=4(j-1)+1}^{m=4(j-1)+4} p_{i, m}^{k}, m=1, \ldots 32 j=1, \ldots 8 .
$$

As a result, each $k$-window produces eight values per channel. The $k$ discrete variable can easily be associated to a specific instant of time, lets say the middle of the $k$-windows. Figure 2 shows the plots of the power values for each frequency band against time. 


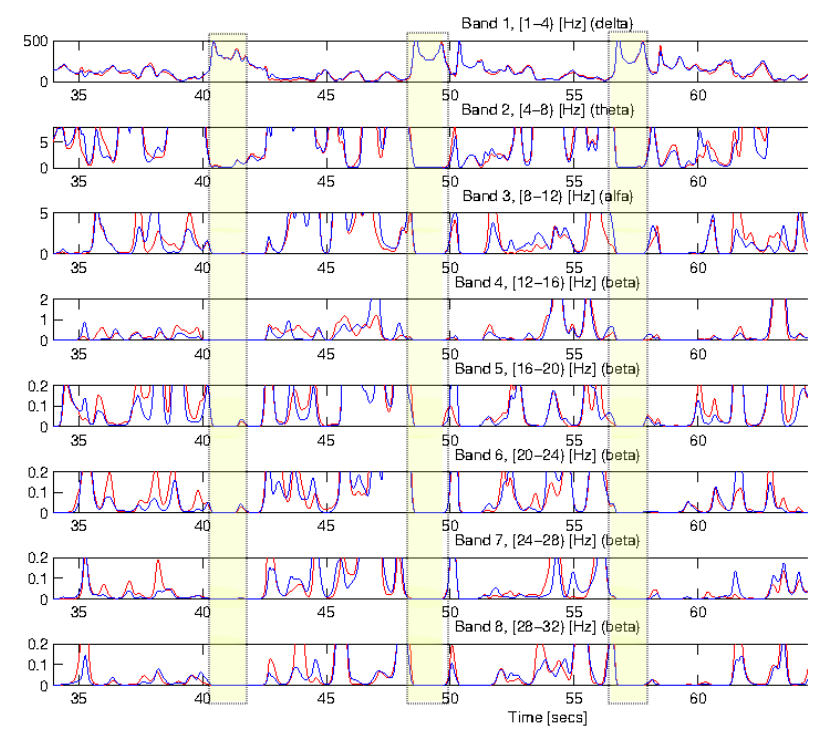

Fig. 2. Spectral power plots for the eight frequency bands

\subsection{Detection of Intent of Movement}

The "intent of movement" detection is important because it allows the signal to be processed, for differentiation purposes, at specific periods of time. When the subject imagines himself performing a movement, some frequency bands are blocked, others are enhanced and some others are synchronized with the signal from the electrode on the opposite side of the brain. An up-flag is turned on if the value of any frequency band is higher than an upper threshold, a down-flag indicates if the value is lower than a bottom threshold and a sync-flag indicates that the difference between two channels is lower than a synchronization threshold. For purposes of robustness, a 36 - sample stabilization window is defined. An event happens, if some of the flags are turned on during the stabilization window. Using this mechanism, a lot of noise associated with short-in-time jumps of the signal are eliminated. Even more, if only certain percentage of the stabilization window is required, not $100 \%$, real mental tasks are not discarded if they do not fully reach the threshold during the complete stabilization window. As a result, each $i$-channel will have blocking $\boldsymbol{b} \boldsymbol{e}_{i}^{k}$, enhancing $\boldsymbol{e}_{i}^{k}$ and synchronizing events $\boldsymbol{s}_{i} \boldsymbol{e}_{i}$, associated to each frequency band as shown in (5).

$$
\begin{gathered}
\left.\boldsymbol{b}_{\boldsymbol{b}} \boldsymbol{e}_{i}^{k}={ }_{b} e_{i, 1}^{k}, \cdots_{b} e_{i, j}^{k}, \cdots_{b} e_{i, 8}^{k}\right] . \\
\left.\boldsymbol{e}_{\boldsymbol{e}} \boldsymbol{e}_{i}^{k}={ }_{{ }_{e}} e_{i, 1}^{k}, \cdots_{e} e_{i, j}^{k}, \cdots_{e} e_{i, 8}^{k}\right] . \\
\left.{ }_{\boldsymbol{s}} \boldsymbol{e}_{i}^{k}={ }_{s} e_{i, 1}^{k}, \cdots_{s} e_{i, j}^{k}, \cdots_{s} e_{i, 8}^{k}\right] .
\end{gathered}
$$


If we are looking for an enhancing event, the upper threshold is relevant while the bottom threshold does not matter, it is set to zero this way is never reached. If we are looking for a blocking event, the bottom threshold is relevant while the upper does not matter and hence it set to infinite (or high enough) to be unreachable. Lets say that zero or infinite thresholds are simply not-relevant thresholds. Each person has characteristic numbers of blocking events $N^{-}$, enhancing events $N^{+}$ and synchronizing events $N^{\sim}$ whereas performs a "mental task". An "intent of movement" is detected if these characteristic numbers $\left(N^{-}, N^{+}, N^{\sim}\right)$ are reached at a specific instant of time.

The length of the interval of time where a mental task are assumed to be performed, varies according to the acquisition protocol between 2 and 8 secs. We presume that a motor-imaginary mental task takes only $0.3 \mathrm{secs}$, hence periods in the signal where the "mental task" is performed are really conformed with two classes of samples, one corresponding to the true mental task realization and other corresponding to relax-time. Thresholds for an specific data set are estimated through an statistical analysis of the signal over mental task realization periods, in comparison with relax-periods.

\subsection{Feature Vector Construction and Classification}

A feature vector is conformed from the spectral power values within a region around $P_{i, j}^{k}$, lets call $P_{i, j}^{k, r}$ the $r$-value in the region. Each $r$ value of spectral power is normalized to obtain $\widetilde{P_{i, j}^{k, r}}$, first the minimum value in the segment is subtracted and subsequently it is divided by the difference between the maximum and the minimum. A gradient vector $\nabla \widetilde{P_{i, j}^{k, r}}$ is estimated using an spectral power difference at every inner point in the segment of the signal (6).

$$
\nabla \widetilde{P_{i, j}^{k, r}}=\left(\widetilde{P_{i, j}^{k, r+1}}-\widetilde{P_{i, j}^{k, r-1}}, t^{r+1}-t^{r-1}\right) .
$$

Notice that the second element of the vector is constant. Instead of that value, the mean of the spectral power differences is used, it allows to enhance differentiation of the angles. A 4-bin histograms with the gradients are built, vectors for every point are grouped according to their angles as follows: bin_1 $=\left[45^{\circ}, 90^{\circ}\right)$, bin_2 $=\left[0^{\circ}, 45^{\circ}\right)$, bin_3 $=\left[-45^{\circ}, 0^{\circ}\right)$ and bin_4 $=\left(-90^{\circ},-45^{\circ}\right)$, subsequently they are counted to conform the histogram. For robustness, the count is weighted with the magnitude of the gradient (7). As a result, a 4-bin histogram for each channel and each band associated to the "intent of movement" is obtained.

$$
\mathbf{H}\left(b_{i n \_} i\right)=\sum_{r}\left|\nabla \widetilde{\mathbf{P}_{r}}\right| \text {, for } r \text { such that } \angle \nabla \widetilde{\mathbf{P}_{r}} \in b i n_{-} i .
$$

The featured vector fed to the classifier $\vec{F}$ is integrated with a selection of the histograms for certain bands of frequency and channels. In the present work, the feature vector was conformed on a 32 -sample segment, using $2^{n} d$ and $6^{t} h$ 
frequency bands and adding the mode and mean values for robustness, the final dimension of the vector is 48. For classification we use a Gaussian classifier (8), we believe that a simple classifier is efficient when the input feature vector is built properly.

$$
p\left(\vec{F} / c_{i}\right)=\frac{1}{(2 \pi)^{\left(\frac{n}{2}\right)}\left|\Sigma_{i}\right|^{\left(\frac{1}{2}\right)}} \exp \left[-\frac{1}{2}\left(\vec{F}-\overrightarrow{\mu_{i}}\right)^{T} \Sigma_{i}^{-1}\left(\vec{F}-\overrightarrow{\mu_{i}}\right)\right] .
$$

\section{Evaluation Data}

The proposed strategy for the EEG pattern recognition was tested with two different input data. First, BCI Competition IV data sets I, provided by the Berlin Group [16. Those data were selected because they have an asynchronous approach, suitable for our objective. In addition, data gathered in our laboratory using the EPOC headset from Emotiv Systems 1 were used too.

\subsection{BCI Competition IV Data Sets I}

EEG data from 59 channels, recorded at a rate of 100 samples per second, from healthy people whereas performing one motor imagery task. The classes of mental task to perform are: (i) imaginary movement of the left hand, (ii) imaginary movement of the right hand and (iii) imaginary movement of any foot. Calibration data were recorded as follows: an arrow was displayed on a computer screen indicating the class of the motor imagery task to perform, the arrow was presented for period of 4 seconds, during which the subject was supposed to imagine the performance of the movement. Periods were interleaved with 2 seconds of blank screen and 2 seconds with a cross in the center of the screen, the cross superimposed to the cue, so it was displayed during 6 seconds.

\subsection{Data Gathered at Our Laboratory}

EEG-data were recorded from the 14 available channels using an EPOC headset from Emotiv System, at a rate of 128 samples per second. Our objective was to gather data with variable time between each mental task realization to simulate self-paced control. We worked with six healthy people, between 20 and 30 years old, each person was asked to select two mental tasks from the following ones: (i) imaginary movement to the right of right hand, (ii) imaginary movement to the left of the left hand, (iii) imaginary movement over the head of both hands and (iv) imaginary movement of the head. In order to get 200 mental task records from each person, five recordings with 40 mental task were performed. A white circle was presented in the middle of a computer screen, it was moved during 2 seconds to indicate the kind of mental task to be performed. The time between each mental task varied between 6 and 10 seconds. Further explanation are available at our Web Page by request. 2

\footnotetext{
${ }^{1}$ Emotiv System, http://emotiv.com/

${ }^{2}$ uamaML datasetI, http://www.eegspiga.com
} 
Table 1. Detection of "intents of movement" and classification for BCI Competition IV dataset I

\begin{tabular}{lccc}
\hline Data set & Detection & rate & Noise Classification rate \\
\hline BCICIV_calib_ds1b & $98 \%$ & 0.18 & $99 \%$ \\
BCICIV_calib_ds1c & $96 \%$ & 0.23 & $97 \%$ \\
BCICIV_calib_ds1d & $92 \%$ & 0.19 & $92 \%$ \\
BCICIV_calib_ds1e & $97 \%$ & 0.21 & $97 \%$ \\
BCICIV_calib_ds1g & $92 \%$ & 0.18 & $98 \%$ \\
uamaML_datasetIb & $76 \%$ & 0.5 & $81 \%$ \\
\hline
\end{tabular}

\section{Experimental Results and Discussion}

Electrodes located over the sensorimotor cortex were taken, according to the 10-10 electrode configuration system. For the BCI Competition IV data, signals in $27^{\text {th }}$ and $31^{\text {st }}$ channels, corresponding to $C 3$ and $C 4$ electrode positions were used in the present work. Similarly, for our own data, signal from $6^{\text {th }}$ and $13^{\text {th }}$ channels, corresponding to $F C 5$ and FC6 electrode positions were used. To evaluate the "intent of movement" detection, a detection rate and an associated noise are calculated from (9) and (10), where TP stands for True Positives, FP for False Positives and $F N$ for False Negatives.

$$
\begin{gathered}
\text { Detection Rate }=\frac{T P}{(T P+F N)} . \\
\text { Noise }=1-\frac{T P}{(T P+F P)} .
\end{gathered}
$$

The proposed strategy detects up to the $98 \%$ of the imagery movements for the five evaluated dataset from the BCI Competition IV data. The noise associated to the false positives is around 0.2 as shown in Tab. 11. The work of Zhang et al. 17. used the same data for self-paced EEG-based motor imagery detection. As a results [17] shown a mean-squared-error of class label prediction for dataset $d s 1 b$ around 0.3 and around 0.23 for dataset $d s 1 g$. For dataset $d s 1 b$ we used $[180,0,0,0,0,0,0,0]$ for upper thresholds and [Inf, 0.2, 0.07, 0.07, 0.007, 0.007, $0.007,0.007]$ for bottom ones, it means that an enhancing-event over band 1 and blocking-events in the other bands were searched. You can see how it is noticeable in Fig. 2, over the dashed areas while the imagery mental task occurs. The classification results using a Gaussian classifier, are close to $99 \%$, leave-oneout evaluated for the BCI Competition IV data, as shown in Tab. 1] Results with our own data reach a detection up to $70 \%$ of "intent of movement" and $80 \%$ of good classification as shown in last row of Tab. 1.

The current system architecture of the prototype is shown in Fig. 3. it includes a MS-Windows Vista PC which receives wirelessly the EEG signal from the EPOC headset at a rate of 128 samples per second. The PC processes data and communicates to a mobile device via Bluetooth. The signal processing programs were developed using Matlab. The client-server Bluetooth application between 


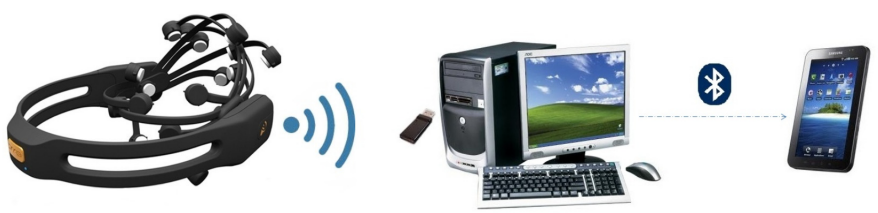

Fig. 3. System architecture of the prototype

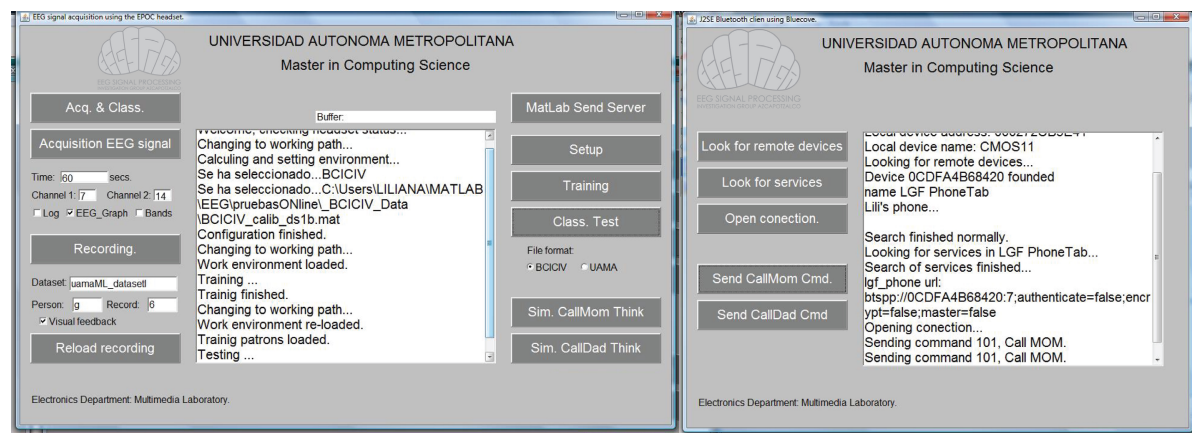

Fig. 4. Application GUI

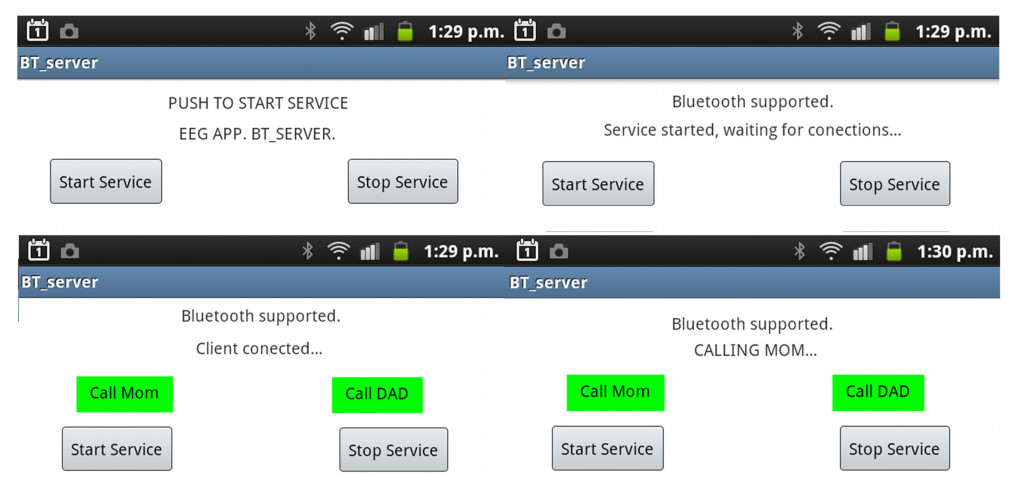

Fig. 5. Screens for the mobile device

the PC and the mobile device was developed using Blue Cove 2.1.0 API JSR-82 implementation library for J2SE 3 and the Bluetooth Android API. 4 The final application has a graphic interface conformed with two windows as shown in Fig. 4. The first one is to work with the EEG signal data: acquisition, training

\footnotetext{
${ }^{3}$ BlueCove 2.1.0 API, http://bluecove.org/bluecove/apidocs/

4 Android Developers:Bluetooth, http://developer .android.com/guide/topics/wireless/bluetooth.html
} 
and using databases files to simulate an input signal. The second one is for the management of Bluetooth connectivity: looking for the remote mobile device, searching for services and establishing connection. Functional evaluation were performed, mental task 1 was associated to the command "call mom" and mental task 2 was associated to "call dad". In the mobile device the server process is started, once the connection is established, when an "intent of movement" is detected and classified, a message is sent to the mobile device in order to make the phone call, as shown in sequence of Fig. 5 .

\section{Conclusions and Further Work}

This work presents a comprehensive point of view for creating a real time control system suitable for mobile devices using EEG signals. Previous works addressed only a particular aspect such as: (i) enabling asynchronous control, (ii) strategy for integrate a feature vector and classification, (iii) final application and (iv) acquisition EEG signal device. Concerning to the EEG signal processing, a phased strategy is proposed, first the detection of an "intent of movement", then the feature vector conformed around the detected instant, as the signal is received and finally the classification. Excellent results were obtained using the BCI Competition IV data sets I. Comparing to related asynchronous works, Borisoff et al. [8] reached up to $80 \%$ of good detection for finger movements and Faradji et al. [9] reached up to $70 \%$ for five mental tasks, some cases with nonoise. Our methodology reached up to $98 \%$. The submitted mental tasks were classified correctly with percentages of classification around $95 \%$ using a simple Gaussian-classifier. Comparing to other methodologies Keirn et al. [10] reached $90 \%$ of good classification, Zhang et. al [12] reached $90 \%$ and Bostanov [14] reached $84 \%$.

Processing remains lightweight and can be carried out in the mobile device. Our vision for future work persues to gather and processes the signal from the mobile. Taking into account the convenience of using a relatively easy and affordable EEG signal acquisition device. Our results are promising, we reached a detection up to $70 \%$ of "intent of movement" and $80 \%$ of good classification. Much work has to be done in regard to the usability of those commercially available devices. Further work comprises a strategy to minimize the noise. Either with a mechanism to detect false positives, avoiding large amount of noise that is sent to the classifier; or by implementing a rejection class that catches all the noise.

Acknowledgments. The authors give thanks to UAM (Universidad Autonoma Metropolitana), ICYTDF (Instituto de Ciencia y Tecnologa del Distrito Federal) and PROMEP under grant UAM-PTC-328 for the economical support. 


\section{References}

1. Pfurtscheller, G., Neuper, C., Schlöl, A., Legger, K.: Separability of EEG signals recordered during right and left motor imagery using adaptive autoregressive parameters. IEEE Transactions on Rehabilitation Engineering 6(3), 316-325 (1998)

2. Müller-Putz, G.R., Scherer, R., Pfurtscheller, G., Neuper, C., Rupp, R.: Noninvasive control of neuroprostheses for upper extremity: Temporal coding of brain patterns. In: EMBS (2009), doi:10.1109/IEMBS.2009.5333185.

3. Tanaka, K., Matsunaga, K., Wang, H.O.: Electroencephalogram-based control of an electric wheel-chair. IEEE Transactions on Robotics 21(4), 762-766 (2005)

4. Chakraborti, T., Sengupta, A., Banerjee, D., Konar, A., Bhattacharyya, S., Khasnobish, A., Janarthanan, R.: Implementation of EEG based control of remote robotic systems. In: International Conference on Recent Trends in Information Systems, pp. 203-208 (2011)

5. De Negueruela, C., Broschart, M., Menon, C., Millan, J.: Brain computer interfaces for space applications. Pers. Ubiquit. Comput. 15, 527-537 (2011)

6. Sajda, P., Pohlmeyer, E., Wang, J., Parra, L.C., Christoforou, C., Dmochowski, J., Hanna, B., Bahlmann, C., Singh, M.K., Chang, S.F.: In a blink of an eye and a switch of a transistor: Cortically coupled computer vision. Proceedings of the IEEE 98(3), 462-478 (2010)

7. Pour, P.A., Gulrez, T., AlZoubi, O., Gargiulo, G., Calvo, R.A.: Brain-Computer Interface: Next generation thought controlled distributed video game development platform. In: CIG, pp. 251-257 (2008)

8. Borisoff, J.F., Mason, S.G., Bashashati, A., Birch, G.E.: Brain computer interface design for asynchronous control applications: Improvements to the LF-ASD asynchronous brain switch. IEEE Trans. on Biomedical Eng. 51(6), 985-992 (2004)

9. Faradji, F., Ward, R.K., Birch, G.E.: A brain computer interface based on mental tasks with a zero false activation rate. In: 4th International IEEE EMBS Conference on Neural Engineering, pp. 355-358 (April-May 2009)

10. Keirn, Z.A., Aunon, J.I.: A new mode of communication between man and his surroundings. IEEE Trans. on Biomedical Engineering 37(12), 1209-1214 (1990)

11. Pfurtscheller, G., Lopes da Silva, F.: Event-related EEG/MEG synchronization and desynchronization: basic principles. Clin. Neuroph. 110, 1842-1857 (1999)

12. Zhang, A., Yang, B., Huang, L.: Feature extraction of EEG signals using power spectral entropy. In: IEEE International Conference on BioMedical Engineering and Informatics, pp. 435-439 (2008)

13. Qin, S., Ji, Z.: Extraction of feature information in EEG signal by virtual EEG instrument with the functions of time-frequency analysis. In: 6th International Symposium on Image and Signal Processing and Analysis, pp. 7-11 (2009)

14. Bostanov, V.: BCI Competition 2003 - Data Sets Ib and IIb: Feature extraction from event-related brain potentials with the continuous wavelet transform and tvalue scalogram. IEEE Trans. on Biomedical Engineering 51(6), 1057-1061 (2004)

15. Sun, S.: Extreme energy difference for feature extraction of EEG signals. Expert System with Applications 37, 4350-4357 (2009)

16. Blankertz, B., Dornhege, G., Krauledat, M., Müller, K.R., Curio, G.: The noninvasive Berlin Brain-Computer Interface: Fast acquisition of effective performance in untrained subjects. NeuroImage 37(2), 539-550 (2007)

17. Zhang, H., Guan, C., Ang, K.K., Wang, C., Chin, Z.Y.: BCI competition IV - data sets I: Learning discriminative patterns for self-paced EEG-based motor imagery detection. Frontiers in Neuroscience 6 (2012), doi:10.3389/fnins.2012.00007 